\title{
Proof of a theorem of the brothers Riesz
}

by

P. KOOSIS (Montpellier)*

1. In this paper we consider functions $F(x)$ of bounded variation defined on some segment or interval (finite or infinite) of the real line. The functions $F^{\prime}(x)$ will always be assumed to be normalized, i. e., $F^{\prime}(x)=\frac{1}{2}[F(x+)+F(x-)]$. If a certain property holds for all $x$ save possibly those belonging to a set $M$ with $\int_{M}\left|d F^{\prime}(x)\right|=0$, we shall say that the property holds a. e. $(d F)$, agreeing that "a. e." used by itself shall mean "almost everywhere with respect to Lebesgue measure".

Our proof of Riesz' theorem shall be based on

THEOREM 1. A necessary and sufficient condition for $F(x)$ to be absolutely continuous is that $F^{\prime}(x)$ exist and be finite a. e. (dF).

The referee has informed me that this theorem is known, being contained in [3], p. 125-128, so we shall restrict ourselves to sketching a proof.

Necessity is evident; for by a theorem of Lebesgue ([4], §11.41), $F^{\prime}(x)$ exists and is finite a. e., hence a. e. $(d F)$ if $F(x)$ is absolutely continuous. As for the sufficiency; if $F^{\prime}(x)$ exists and is finite a. e. $(d F)$, a theorem of Egoroff ([4], p. 339) shows that for any $\varepsilon>0$ there exist a $C<\infty$, a compact set $M$, and an $h_{0}>0$ such that $\int_{M^{\prime}}\left|d F^{\prime}(x)\right|<\varepsilon, M^{\prime}$ being the complement of $M$, whilst $\left|h^{-1}\left[F(x+h)-F^{\prime}(x)\right]\right|<C, x \in M$, $|h|<h_{0}$. It then follows almost immediately, that if $E$ is any closed subset of $M$ with Lebesgue measure zero, $\int_{E} d F(x)=0$. (The argument is obvious; one uses finite coverings of the compact set $E$ by disjoint intervals and the simplest properties of Lebesgue-Stieltjes integrals). Thus, if $D$ is any closed set of Lebesgue measure zero, $\left|\int_{D} d F(x)\right|$ $\leqslant\left|\int_{D} \int_{M} d F^{\prime}(x)\right|+\left|\int_{D \sim M^{\prime}} d F^{\prime}(x)\right|<\varepsilon$, or, since $\varepsilon$ is arbitrary, $\int_{D} d F^{\prime}(x)=0$. This last demonstrates absolute continuity of $F^{\prime}(x)$.

Remark. The same argument shows, more generally, that $F(x)$ is absolutely continuous on any set where its derivative exists a. e. (dF) and is finite.

* Fulbright scholar. 
2. Lemma. Let $V(x)$ be non-decreasing. $V^{\prime}\left(x_{i}\right)$ exists, finite or infinite, a.e. $(d V)$.

(This holds with $V(x)$ replaced by any function $F(x)$ of bounded variation: for if $V(x)$ denotes the total variation of $F(x), d F(x) / d V(x)$ exists and has absolute value one a. e. $(d V)$.)

Proof. The function $Y(x)=V(x)+x$ is strictly increasing; let $X(y)=\inf \{x: Y(x)>y\} . X(y)$ is non-decreasing, whence $X^{\prime}(y)$ exists a. e.; that is, on the set of points of continuity of $V(x),\left(V^{\prime}(x)+1\right)^{-1}$ $=X^{\prime}(Y(x))$ exists a. e. $(d Y)$, hence a. e. $(d V)$. On the other hand, $V^{\prime}(x)$ clearly exists and equals $\infty$ at each point of discontinuity of $V(x)$.

THEOREM 2. (of F. and M. Riesz; see [5], p. 158, [2], and [1]). Let $F(\theta)$ be of bounded variation on $[-\pi, \pi]$ and of period $2 \pi$. If

$$
f\left(r e^{i \varphi}\right)=\frac{1}{2 \pi} \int_{-\pi}^{\pi} \frac{1-r^{2}}{1+r^{2}-2 r \cos (\varphi-\theta)} d F^{\prime}(\theta)
$$

is analytic in $0 \leqslant r<1, H^{\prime}(\theta)$ is absolutely continuous.

Proof. Let $V(\theta)$ be the total variation of $F(\theta)$; it is enough to show that $V(\theta)$ is absolutely continuous on $(-\pi, \pi)$. For one may then apply the same result to $F^{\prime}(\theta+\pi / 2)$ and conclude that $F^{\prime}(\theta)$ is absolutely continuous everywhere, including at $\pi$ and $-\pi$. Form

$$
u\left(r e^{i \varphi}\right)=\frac{1}{2 \pi} \int_{-\pi}^{\pi} \frac{1-r^{2}}{1+r^{2}-2 r \cos (\varphi-\theta)} d V(\theta) ;
$$

let $v\left(r e^{i \varphi}\right)$ be the harmonic conjugate of $u\left(r e^{i p}\right)$ in $0 \leqslant r<1$, and let $g(z)=u(z)+i v(z)$. In $|z|<1, g(z)$ is regular and $\chi g(z)=u(z)>0$ since $V(\theta)$ is non-decreasing.

Now by the lemma, $V^{\prime}(\theta)$ exists a. e. $(d V)$ in $(-\pi, \pi)$, finite or infinite. Let $E=\left\{\theta ;-\pi<\theta<\pi, V^{\prime}(\theta)=\infty\right\}$, and let $E^{\prime}$ be the complement of $E$ in $(-\pi, \pi)$.

(i) For $\theta \in E, \lim _{r \rightarrow 1} u\left(r e^{i \theta}\right)=\infty$, by a theorem of Fatou ([2], p. 52), whence $g\left(r e^{i \theta}\right) \rightarrow \infty$ in the right-half plane as $r \rightarrow 1$.

(ii) Since $R g(z)>0,\left(1+g\left(r e^{i \theta}\right)\right)^{-1}$ is bounded, $0 \leqslant r<1$, therefore, by onother theorem of Fatou ([2], p. 145-146), this last tends to a limit for almost all $\theta$ as $r \rightarrow 1$. In particular, $\lim g\left(r e^{i \theta}\right)$ exists a. $\theta$. on $E^{\prime}$, finite or infinite. By the lemma, $\nabla^{\prime}(\theta)$ is finite a. e. $(d V)$ on $E^{\prime}$, and the remark of $\S 1$ shows that a property which holds a. e. on $E^{\prime}$ holds there a. e. $(d V)$; this is thus the case with the limit under consideration.
In fine, $\lim g\left(r e^{i \theta}\right)$ exists a. e. $(d V)$ on $(-\pi, \pi)$, finite or infinite.

Assume that $\stackrel{r \rightarrow 1}{V}(\theta)$ is not absolutely continuous on $(-\pi, \pi)$.

Then, if $\Omega=\left\{\theta ;-\pi<\theta<\pi, \lim g\left(r e^{i \theta}\right)=\infty\right\}$, we have $E \subseteq \Omega$ by (i), and the lemma, together with theorem 1 , implies that $\int_{\Omega} d V(\theta)>0$. Or, since $V(\theta)$ is the total variation of $F(\theta)$, we can find an interval $J \subset(-\pi, \pi)$ with $\int_{\Omega^{\prime}} e^{i \theta} d F(\theta) \neq 0$, where $\Omega^{\prime}=\Omega \cap J$. The limit of $f\left(r e^{i \theta}\right)$ (defined in the hypothesis) as $r \rightarrow 1$ exists and is finite a. e., (since $H^{\prime}(\theta)$ exists and is finite a. e., see [3], p. 53), so that we may suppose $J$ so chosen that this holds when $\theta$ is either of its endpoints. If $J=(\alpha, \beta)$, we let $C$ be the contour, described in the positive sense, which consists of the two radii $r e^{i \alpha}, r e^{i \beta}, 0 \leqslant r<1$, and the are $e^{i \theta}, \alpha \leqslant \theta \leqslant \beta$.

On $O$ we define a measure $d m(z)$ as follows:

$$
\begin{gathered}
d m(z)=e^{i \alpha} f\left(r e^{i \alpha}\right) d r \quad \text { for } \quad z=r e^{i \alpha}, 0 \leqslant r<1, \\
d m(z)=i e^{i \theta} d F(\theta) \quad \text { for } \quad z=e^{i \theta}, a \leqslant \theta \leqslant \beta, \\
d m(z)=-e^{i \beta} f\left(r e^{i \beta}\right) d r \quad \text { for } \quad z=r e^{i \beta} 0 \leqslant r<1 .
\end{gathered}
$$

It is then clear that $\int_{C}|d m(z)|<\infty$. Moreover, if $\Psi(z)$ is analytic in a region including the closed interior of $C$, we have $\int_{C} \Psi(z) d m(z)=0$, this being an obvious consequence of the analyticity of $f(z)$ in $|z|<1$, Canchy's theorem, and the fact that

$$
\int_{a}^{\beta} \Psi\left(r e^{i \theta}\right) i r e^{i \theta} f\left(r e^{i \theta}\right) d \theta \rightarrow \int_{\alpha}^{\beta} \Psi\left(e^{i \theta}\right) i e^{i \theta} d F^{\prime}(\theta) \quad \text { as } \quad r \rightarrow 1 .
$$

Finally, let $\Phi(w)=w(1+w)^{-1} ; \Phi(w)$ is anclytic and in absolute value $<1$ in $R w>0$, and can be continuously extended there so as to mop $\infty$ into the point 1 . Since $R g(z)>0$ for $|z|<1, \Phi(g(r z))$ is, for $r<1$, analytic in a region properly including $C$. Thus, $\int \Phi(g(r z)) d m(z)=0$. But, by the above discussion, $\lim _{r \rightarrow 1} g(r z)$ exists a. e. $(d m)$ on $C$, finite or infinite, whilst $|\Phi(g(r z))|$ remians $<1$ on $O$ as $r \rightarrow 1$. Hence, if we define $g\left(e^{i \theta}\right)=\lim _{r \rightarrow 1} g\left(r e^{i \theta}\right)$ we have, by Lebesgue's bounded convergence theorem,

$$
\int_{\sigma} \Phi(g(z)) d m(z)=0,
$$

since clearly $\Phi(g(z))=\lim _{r \rightarrow 1} \Phi(g(r z)), z \epsilon C, \Phi(\infty)$ having been defined to equal 1. And the same is true with $\Phi(g(z))$ replaced by its $n^{\text {th }}$ power for $n=2,3,4, \ldots$ But for $z \epsilon C, g(z)=\infty$ if $z$ is of the form $e^{i \theta}, \theta \epsilon \Omega^{\prime}$, and otherwise $|g(z)|<\infty$ a. e. $(d m)$; that is, $\Phi(g(z))=1$ or is in absolute 
value $<1$, according as $z=e^{i \theta}$ with $\theta \epsilon \Omega^{\prime}$ or not. We may thus apply Lebesgue's bounded convergence theorem once more to obtain

$$
0=\int_{\sigma} \lim _{n \rightarrow \infty}\left[\Phi((g(z))]^{n} d m(z)=\int_{\Omega^{\prime}} i e^{i \theta} d F^{\prime}(\theta),\right.
$$

contardicting $\int_{\Omega^{\prime}} e^{i \theta} d F^{\prime}(\theta) \neq 0$. The proof is complete.

\section{Multiplikatoren für starke Konvergenz von Fourierreihen I}

\section{References}

[1] H. Helson, On a theorem of F. and M. Riesz, Coll. Math. 3 (1955), p. 113 - 117 [2] F. and M. Riesz, Über die Randwerte einer analytischen Frunltion, IV Cong des math. scand. 1916, p. 27-44.

[3] S. Saks, Theory of the integral, Warszawa 1937.
[4] E. C. Titchmarsh, The theory of functions, Oxford 1939.
[5] A. Zygmund, Trigonometrical series, Warsawa 1935.

Regu par la Rédaction le 23.1. 1958
G. GOES (Ludwigsburg)

1. Einleitung und Definitionen. Herr Karamata [7] bewies folgenden Satz:

Die reellen Multiplikatoren $\left\{\lambda_{j}\right\}(j=0,1,2, \ldots)$ transformieren die Fourierreihe

$$
\frac{a_{0}}{2}+\sum_{j=1}^{\infty}\left(a_{j} \cos j t+b_{j} \sin j t\right)
$$

jeder stetigen Funktion genau dann in eine gleichmäßig konvergente Fourierreihe.

$$
\frac{\lambda_{0} a_{0}}{2}+\sum_{j=1}^{\infty} \lambda_{j}\left(a_{j} \cos j t+b_{j} \sin j t\right)
$$

wern

$$
\int_{0}^{\pi}\left|\frac{\lambda_{0}}{2}+\sum_{j=1}^{n} \lambda_{j} \cos j t\right| d t=O(1) \quad \text { für } \quad n \rightarrow \infty .
$$

Die Absicht der hier vorliegenden Note ist es, verwandte Sätze für Multiplikatoren zwischen vcrschiedenen linearen und normierten Räumen zu beweisen. Der oben genannte Satz von Karamata ist als Spezialfall in unserem Satz 1 enthalten.

Unsere Sätze 1 bis 3 stellen eine Erweiterung beknanter Sätze über Multiplikatoren dar, die aus zahlreichen Arbeiten, vorwiegend polnischer Mathematiker, bekannt sind. Wir nennen W.H. Young (1912, 1913), H. Steinhaus $(1916,1919,1926,1929)$, S. Szidon (1921, 1939), M. Fekete (1923), A. Zygmund $(1927,1935)$, S. Bochner (1929), W. Orlicz (1929 bis 1954), S. Kaczmarz (1933, 1938), J. Marcinkiewicz (1938, 1939), S. Verblunsky $(1932,1935)$, L. B. Hedge (1943) und G. A. Alexits (1951). Die Erweiterung besteht darin, daß als Bildraum $E_{1}$ Funktionenräume genommen werden, in denen die erzeugten Fourierreihen stark, das heißt nach der Norm des jeweiligen Raumes, konvergieren. 